\title{
Study the effect of education based on the theory of planned behavior in the prevention of AIDS among addicts
}

Alireza Abbaspour

MSc. Dept. of Health Education and Promotion, Science and Research Branch, Islamic Azad University, Tehran, Iran

Zahra Jalili

* Associate Professor, Dept. of Health Education and Promotion, Science and Research Branch, Islamic Azad University, Tehran, Iran. (Corresponding Author) zahra.jalili@gmail.com

Davoud Shojaeizadeh

Professor, Dept. of Health Education and Promotion, Science and Research Branch, Islamic Azad University, Tehran, Iran.

\section{Received: 2021/01/24}

Accepted: 2021/04/18

Doi: 10.52547/ijhehp.9.2.201

\begin{abstract}
Background and Objective: Addicts are one of the carriers of HIV/AIDS who should be trained to prevent this transmission. Due to the importance of education in the prevention of HIV / AIDS, the present study was conducted to investigate the effect of education based on the theory of planned behavior in the prevention of AIDS. Materials and Methods: This quasi-experimental study was performed in 20192020 on 100 addicts referred to addiction treatment centers (DIC) in Sari who were selected by convenience sampling method. Data were gathered in two phases (before and two months after implementing the intervention) using a designed questionnaire to measure the Theory of Planned Behavior (TPB) constructs fill for both groups. The educational intervention was designed in four educational sessions (one-hour) according to the results obtained in the pre-test and was performed for the intervention group. Data were analyzed using the statistical tests in the SPSS-23.

Results: Before the intervention, there was no significant difference in the structures of the theory of planned behavior between the two groups ( $p>0.05)$, but after the intervention, attitude scores from $18.12 \pm 4.96$ to $21.92 \pm 6.62$, abstract norms from $19.38 \pm 3.62$ to $22.66 \pm 5.38$, perceived behavioral control from $17.85 \pm 4.92$ to $20.61 \pm 6.13$, behavioral intention from $12.42 \pm 4.62$ to $15.44 \pm 6.65$ and behavior from $12.40 \pm 4.34$ to $16.38 \pm 6.47$ in the experimental group $(p<0.05$ ).

Conclusion: The results of the present study showed that the educational intervention based on the theory of planned behavior is effective in preventive behaviors of AIDS in addicts. Due to the significant effect of the intervention on the structures of the theory of planned behavior, education based on this theory can be used as a framework for designing and implementing educational interventions to prevent HIV/AIDS among addicts.
\end{abstract}

Keywords: AIDS, HIV Infections, Drug Addiction, Theory of Planned Behavior, Intervention

Paper Type: Research Article.

Citation (Vancouver): Abbaspour A, Jalili Z, Shojaeizadeh D. Study the effect of education based on the theory of planned behavior in the prevention of AIDS among addicts. Iran J Health Educ Health Promot. Summer 2021;9(2): 201-211.

Citation (APA): Abbaspour A., Jalili Z., Shojaeizadeh D. (Summer 2021). Study the effect of education based on the theory of planned behavior in the prevention of AIDS among addicts. Iranian Journal of Health Education \& Health Promotion., 9(2), 201-211. 


\section{بررسى تاثيرآموزش مبتنى بر نظريه رفتار برنامهريزى شده در بيشغيرى از \\ بيمارى ايدز در بين معتادان}

\section{جكيده}

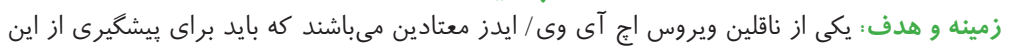

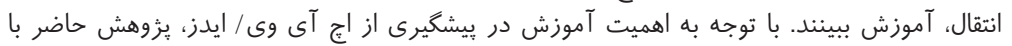

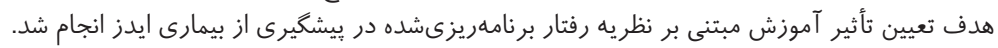

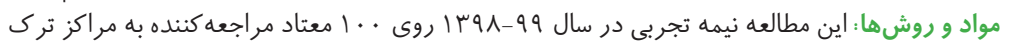

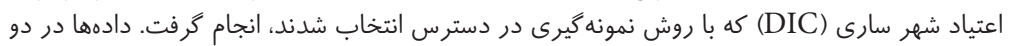

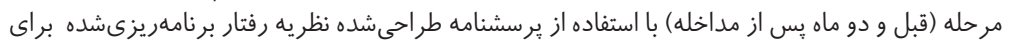

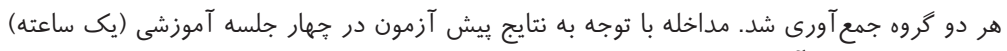

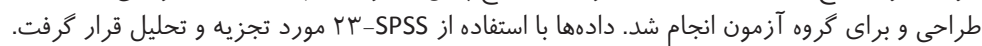

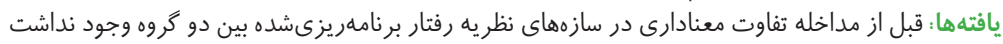

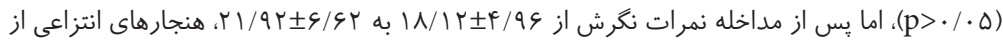
رو رفتارى از إن

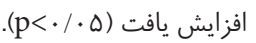

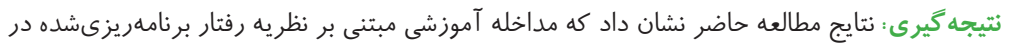

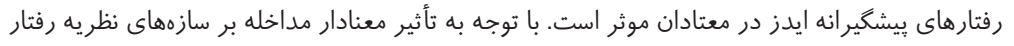

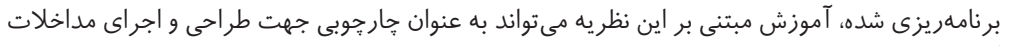

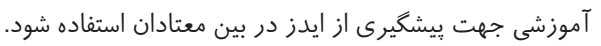

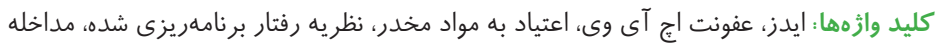
نوع مقاله : مطالعه يزوهشى.

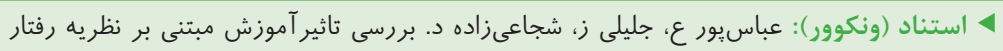

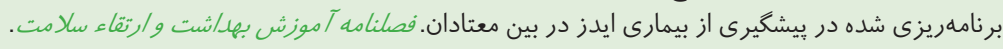

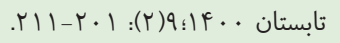

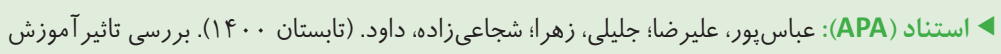

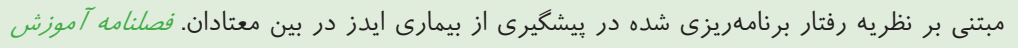

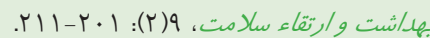

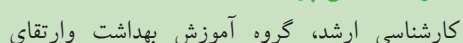

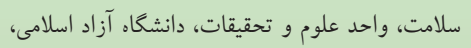

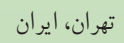

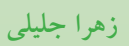
* مانشيار كروه آموزش بهداشت و ارتقاى سلامت، واحد علوم و تحقيقات، دانشكاه آزاد اسلامى، تهران، zahra.jalili@gmail.com

$$
\text { ايران. (نويسنده مسئول) }
$$

داود شجاعى زاده استاد كروه آموزش بهداشت و ارتقاى سلامت، واحد آداد

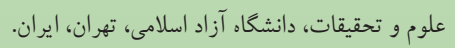

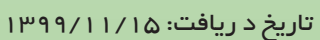

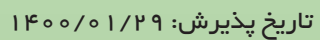


مِرخطر، انتقال از راههاى مختلف و متنوع، نبودن واكسن، عدم

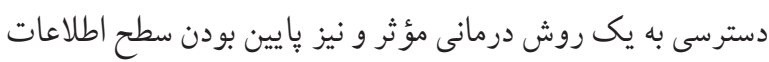

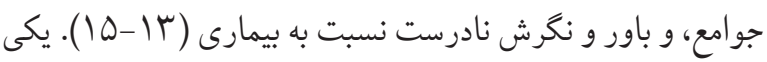

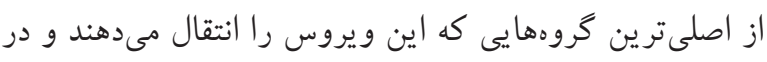

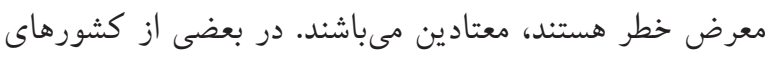

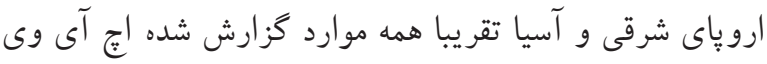

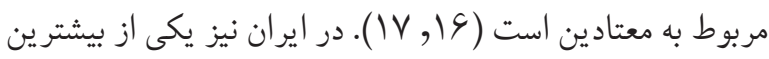

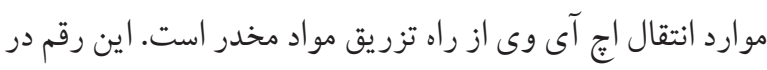

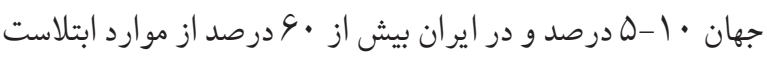

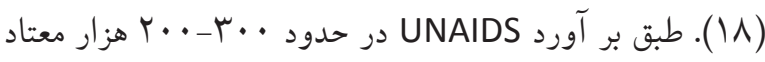

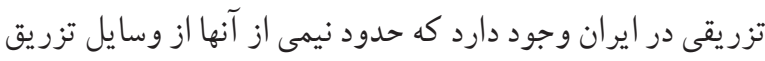
مشتر ك استفاده مى كنند (19).

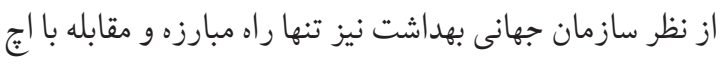

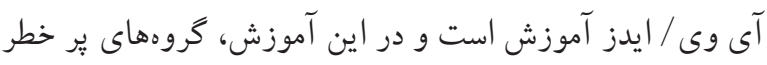

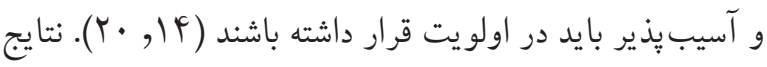

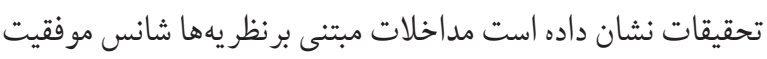

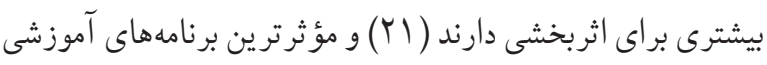

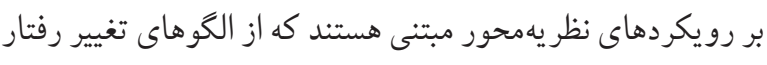

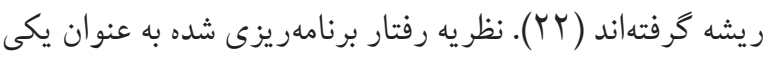

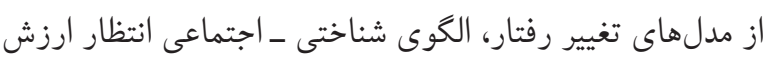

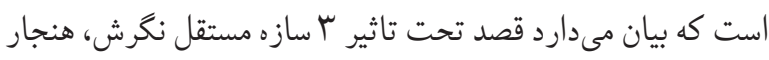

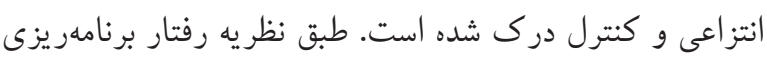

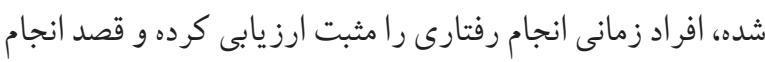

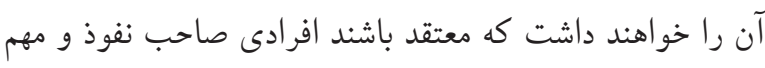

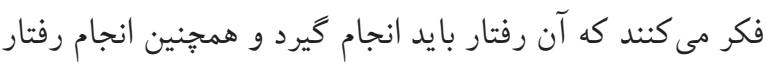

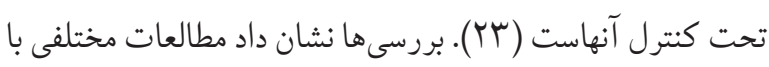

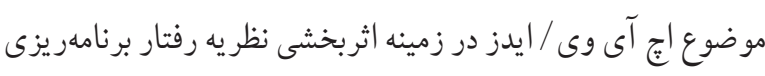

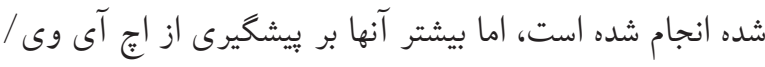

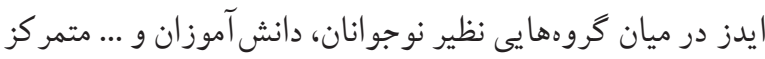

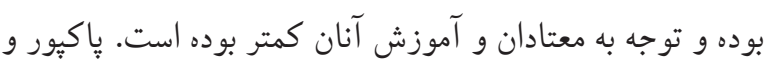

اججى آىوى' ويروس نقص ايمنى انسانى است كه به دستكاه ايمنى بدن

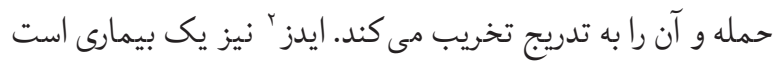

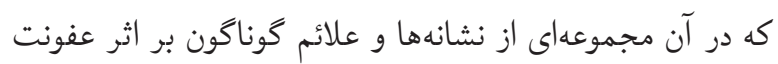

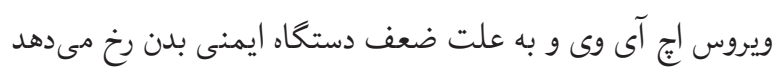

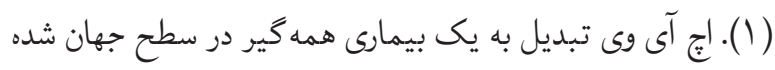

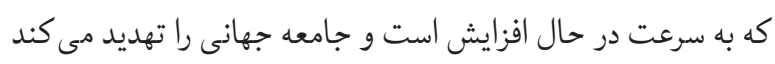

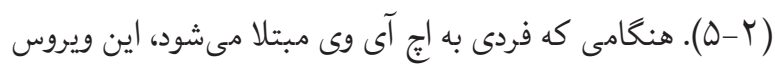

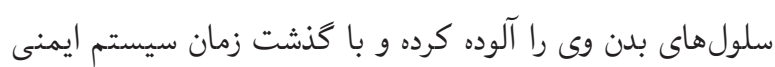

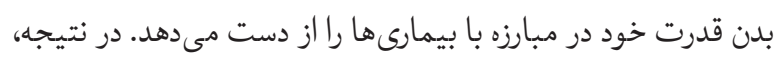
ميكروب هايى كه در يك بدن سالم مى تواند به راحتى نابود شود، أج

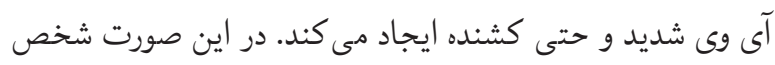
وارد مرحله بيمارى ايدز شده است (9) (9).

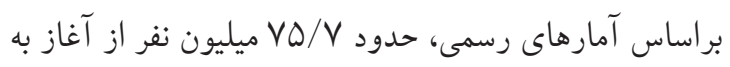

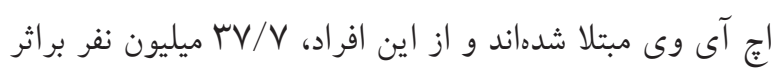

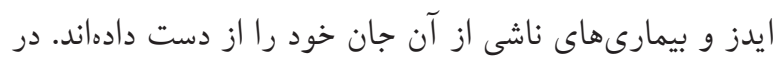

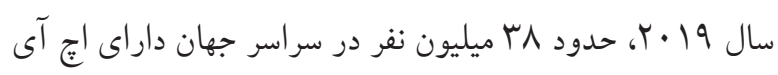

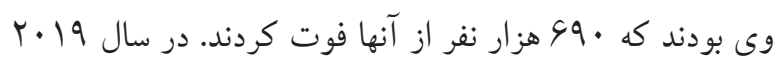

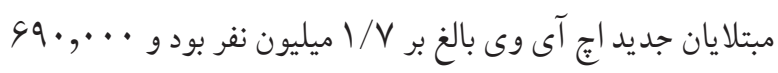

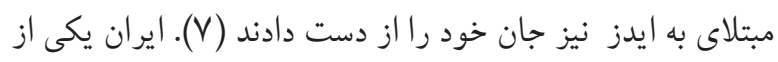

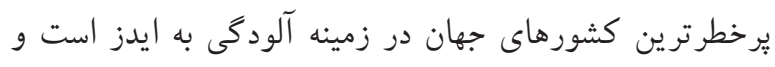

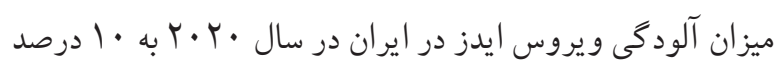

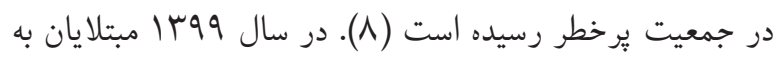

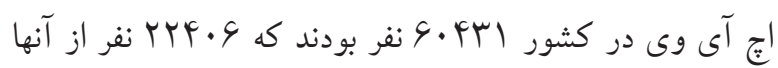

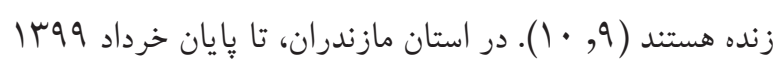

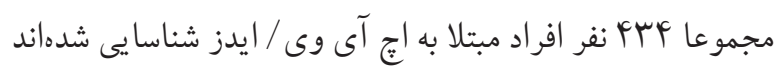

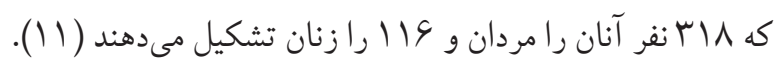

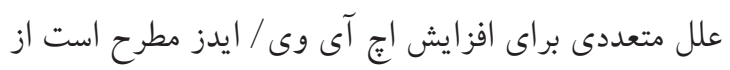

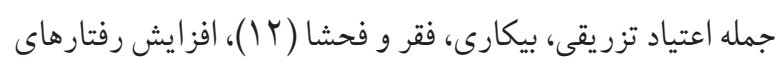


بيمارى، عدم تمايل به ادامه درمان با متادون و زندان بود.

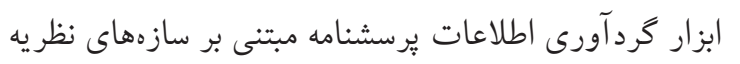

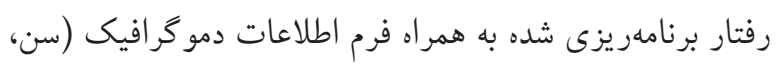

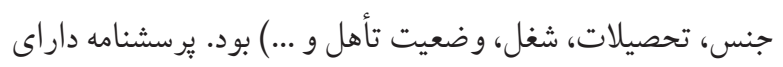

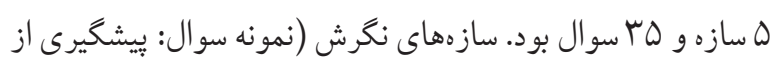

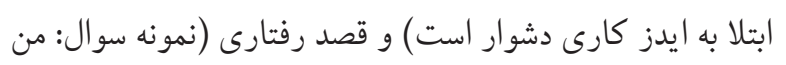
قصد دارم از وسايل مشترك نظير سرنح و تيغ و ... استفاده نكنم) براساس طيف ليكرت ه كزينهاى كاملا مخالفم (نمره ()، مخالفم

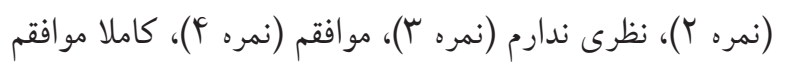

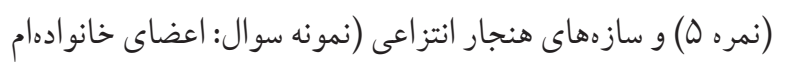

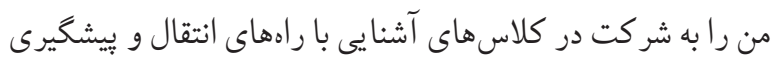
از ايدز تشويق مى كنند)، كنترل رفتارى درى شده (نمونه سوال:

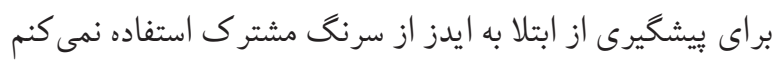

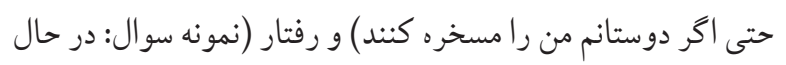

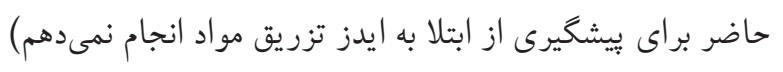

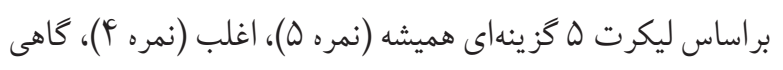

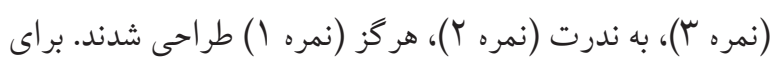

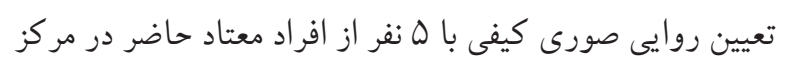

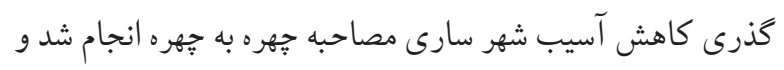
دشوارى درى سو الها و كلمات، تناسب و ارتباط سوالها با با ابعاد

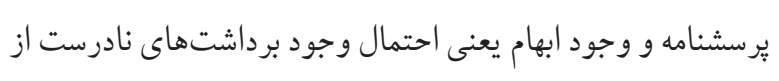

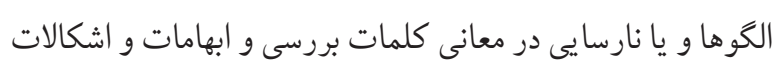
موجود اصلاح گرديد. براى تعيين روايى صورى كمى از روش (انمره تأثير) جهت كاهش و حذف سوالهاى نامتناسب و و تعيين اهميت

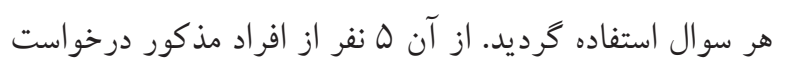

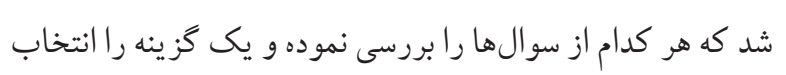

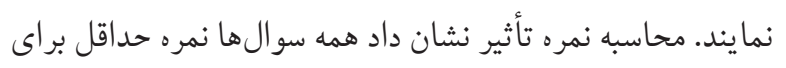

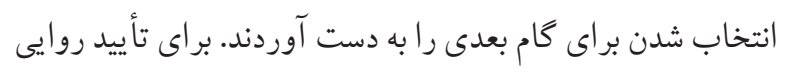

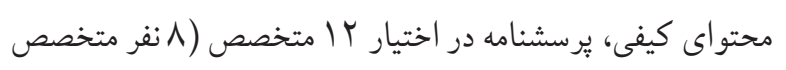

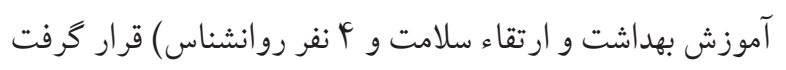
تا بِ از مطالعه دقيق، ديد كاههاى اصلاحى خود در زمينه رعايت
همكاران نشان دادند آموزش بر اساس نظريه رفتار برنامهر يزىشده

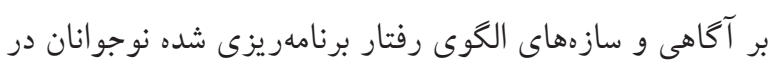

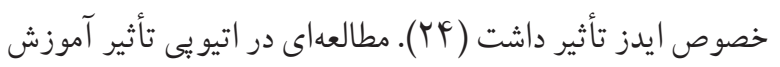

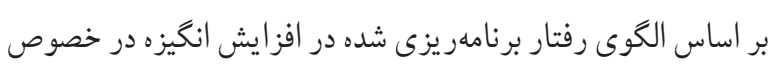

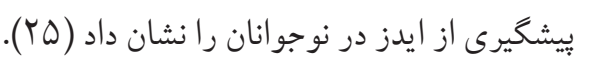

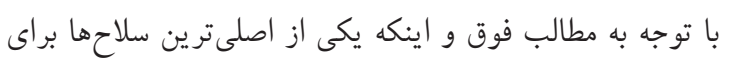

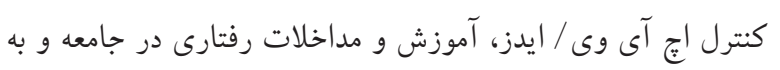

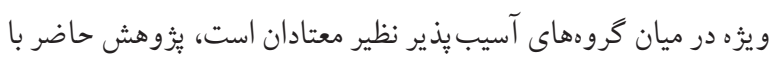

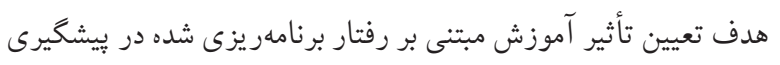

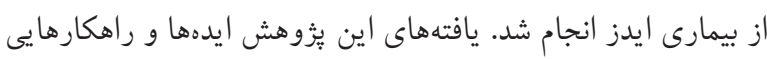

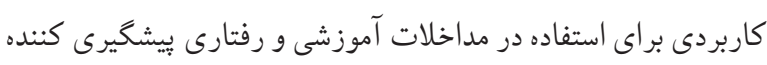

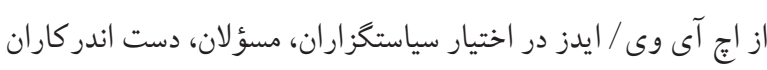
و فعالان ذىربط قرار مىدهد. روش بررسى يزوهش حاضر يك مطالعه مداخلهاى از نوع نيمه تجربى قبل و بعد بود. جامعه آمارى يُزوهش حاضر مركز گذرىى كاهش آسيب شهر سارى در سال 99-1911 (DIC)

$$
N=\frac{\left(\frac{1}{96}+\frac{0}{84}\right)^{2}\left(2 S^{2}\right)}{\frac{0}{36 \square}^{2}}
$$
زير بدست آمد. تعداد نمونه FF أنفر در هر گروه به دست آمد كه با احتساب ريزش

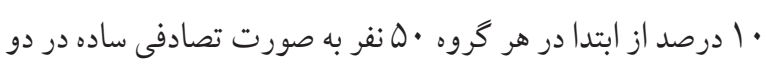

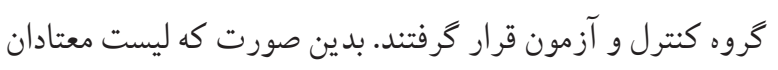

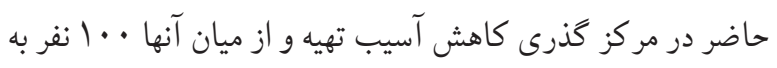

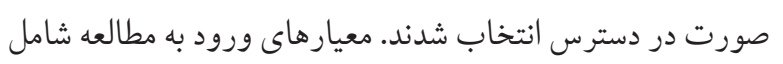

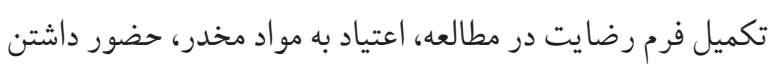
در مركز خذرى كاهش آسيب و مرد بودن بود. معيارهاى خروج

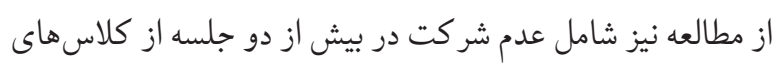
آموزشى، ترخيص از مركز كذرى كاهش آسيب به دليل مهاجرت، 
تحقيات تهران (IR.IAU.SRB.REC.1399.126) و انجام هماهنكى با مسئولان دانشكاه و اخذ مجوز و معرفىنامه، نمونهها انتخاب و

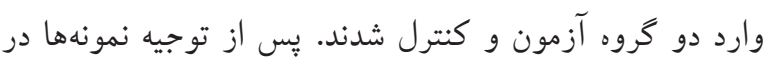

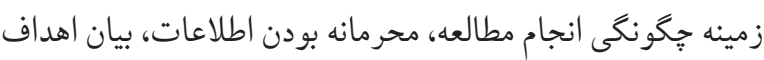
مطالعه، و كسب رضايت كتبى از آنها، مطالعه آغاز شد. اطلاعات

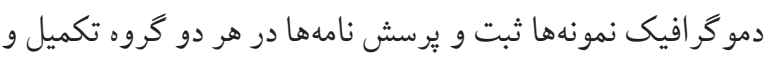

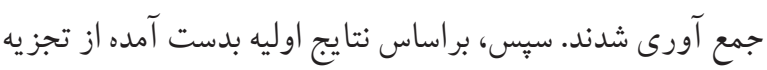

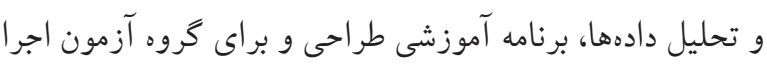

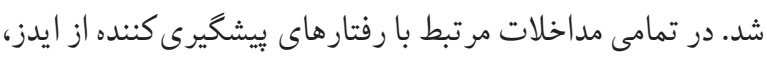

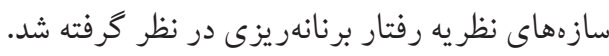

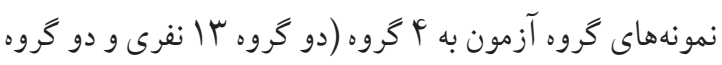

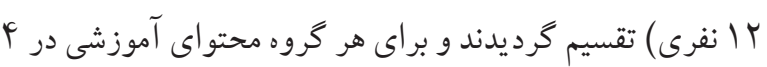

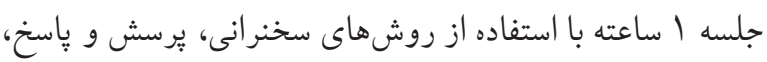

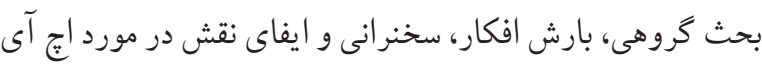

وى / ايدز اجراشد (جدول ()).
دستور زبان، استفاده از كلمات مناسب، قرار كيرى سوالها در جاى مناسب خود، و امتيازدهى مناسب را به صورت مبسوط و كتبى ارائه

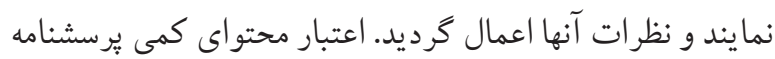

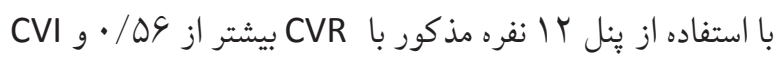

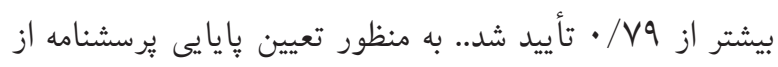

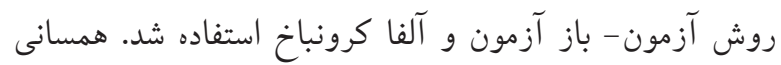

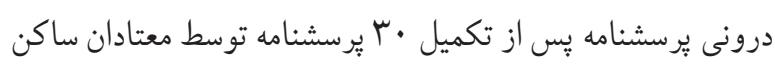

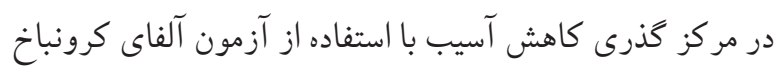

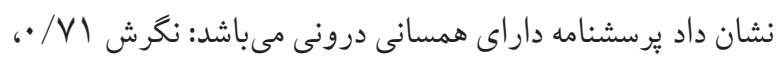

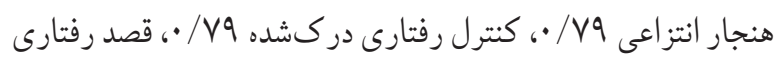

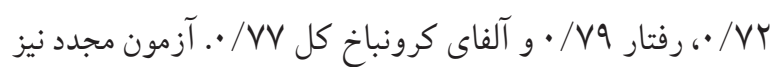

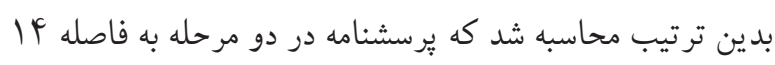

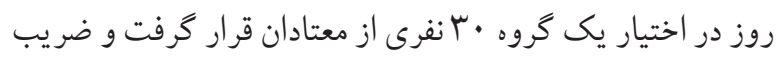

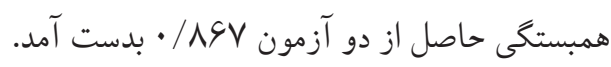

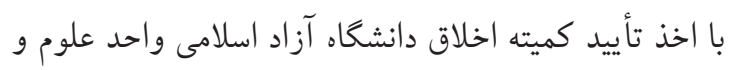

جدول ا. فعاليتهاى آموزشى و جدول زمانبندى جلسات آموزشى در كَروه آزمون

\begin{tabular}{|c|c|c|c|c|}
\hline استر اتخىها & آموزشدهنده & 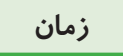 & 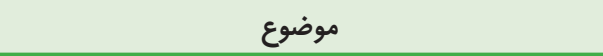 & جلسه \\
\hline سخنرانى، برسش و پِاسخ & محقق & 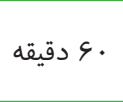 & 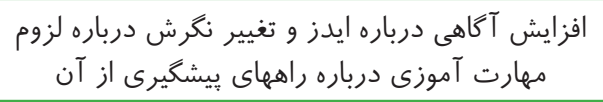 & اول \\
\hline سخنرانى و بارش افكار & محقق & 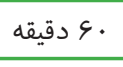 & تأثير گذارى بر سازه كنترل رفتارى درى شده & دوم \\
\hline سخنر انى و ايفاى نقش & محقق، يك معتاد و يك نفر از & 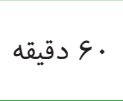 & تأثير گذارى بر سازه هنجار انتزاعى & سوم \\
\hline سخنرانى، يرسش و پاسخ، & محقق و يك معتاد جهت كمك آموزش & 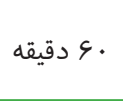 & آشنايى فراگيران با مهارت ((نه) كفتن با تأثير بر سازه & 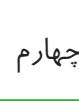 \\
\hline
\end{tabular}

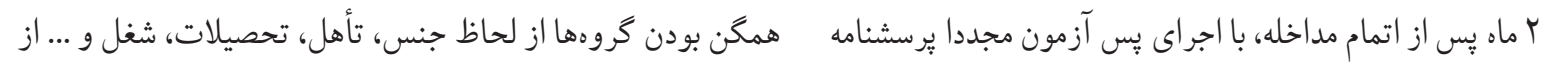

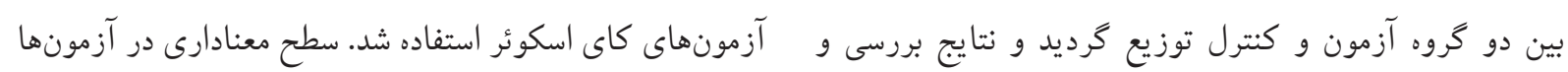

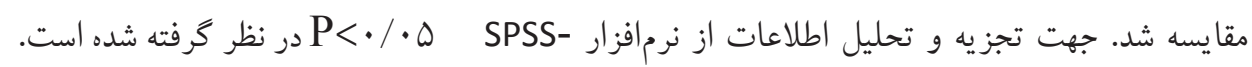

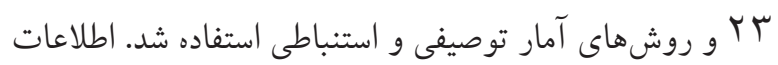

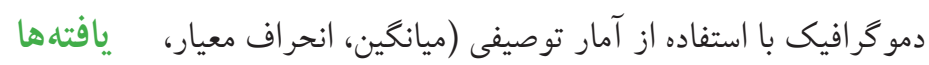

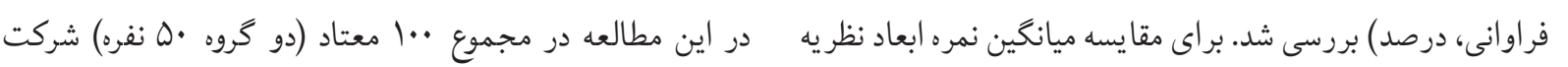

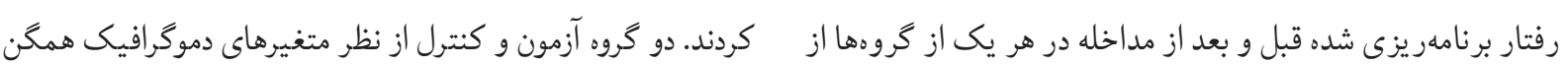

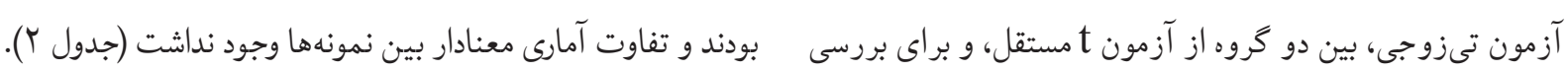


جدول r. مقايسه متغيرهاى دموكرافيك در دو گروه كنترل و آزمون

\begin{tabular}{|c|c|c|c|c|}
\hline P-value ${ }^{a}$ & Sروه كنترل (\%) & يروه آزمون (\%) & طبقات & متغير \\
\hline \multirow{5}{*}{$\cdot / \mu F$} & $(r) 1$ & - & كمتر از • r سال & \multirow{5}{*}{ سن } \\
\hline & $(I r) G$ & $(1 \cdot) \Delta$ & •r تا • r سال & \\
\hline & $(F r) r r$ & $(\Delta \cdot) r \Delta$ & 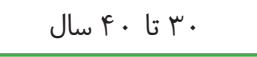 & \\
\hline & $\left(K^{w}\right) \mid V$ & (צr) & 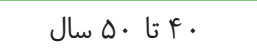 & \\
\hline & $(\Lambda) F$ & $(\Lambda) F$ & بيشتر از •0 سال & \\
\hline \multirow{4}{*}{$\cdot / V S$} & (sq) & I & زير دييلم & \multirow{4}{*}{ سطح تحصيلات } \\
\hline & r & $(r \wedge) \mid r$ & ديثلم & \\
\hline & $(1 \cdot) \Delta$ & $(\Lambda) F$ & فوق ديهلم و ليسانس & \\
\hline & - & $(Y) 1$ & بالاتر از ليسانس & \\
\hline \multirow{3}{*}{$\cdot / 10$} & $(\zeta \wedge) \mu r$ & $(g F) r r$ & متأهل & \multirow{3}{*}{ وضعيت تاهل } \\
\hline & $(11) 9$ & $(1 \wedge) 9$ & مجرد (ازدواج نكرده) & \\
\hline & $(\mid f) V$ & $(1 \wedge) 9$ & طلاق گرفته & \\
\hline \multirow{4}{*}{.109} & $(\Delta \wedge) r q$ & $(\Delta F) Y V$ & شاغل & \multirow{4}{*}{ وضعيت اشتغال } \\
\hline & (G) & $(r \wedge) \mid F$ & بيكار & \\
\hline & - & $(Y) 1$ & بازنشسته & \\
\hline & $(1 \cdot) \Delta$ & $(1 \varepsilon) \wedge$ & ساير & \\
\hline \multirow{4}{*}{.$/ 09$} & $(r \cdot) r$. & $(Y \mathcal{F}) \mid V$ & كمتر از 1 ميليون & \multirow{4}{*}{ خيزان درآمد ماهيانه } \\
\hline & $(\mu F) \mid V$ & $(\mu F) \mid V$ & ا تا r ميليون & \\
\hline & $(1 \varepsilon) \wedge$ & س & r تا ب ميليون & \\
\hline & $(1 \cdot) \Delta$ & $(c) \mu$ & بيشتر از ب ميليون & \\
\hline \multirow{5}{*}{.191} & $(\mid f) V$ & $(1 \cdot) 0$ & كمتر از ه سال & \multirow{5}{*}{ مدت زمان مصرف مخدر } \\
\hline & $(19) \wedge$ & $(Y Y) \|$ & ه تا • ا سال & \\
\hline & $(\mu \cdot) 10$ & r & •ا تا ها سال & \\
\hline & $(r \cdot) 1 \cdot$ & $(r \cdot))$ & ها تا • r سال & \\
\hline & $(r \cdot) 1 \cdot$ & $(Y Y) \backslash 1$ & بيشتر از · r سال & \\
\hline \multirow{2}{*}{$\cdot / \Delta F$} & $(\Delta r) r G$ & r & بله & \multirow{2}{*}{ سابقه زندان } \\
\hline & $(F \wedge) r F$ & $(\Delta F) Y V$ & خير & \\
\hline \multirow{2}{*}{$\cdot / \Delta r$} & $(I r) s$ & $(19) \wedge$ & بله & \multirow{2}{*}{ داشتن دوستان مبتلا } \\
\hline & $(\Lambda ૬)$ Fr & $(\Lambda F) F r$ & خير & \\
\hline
\end{tabular}

a: Chi-Square

نتايج آزمون تىمستقل نشان داد. بين دو گروه كنترل و اما اين تفاوتها بين دو گروه در مرحله بعد از مداخله آموزشى

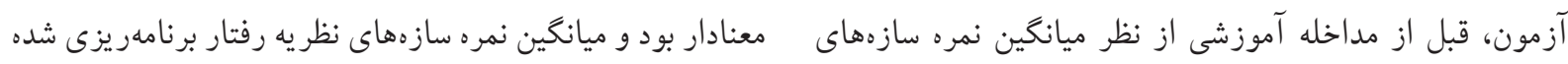

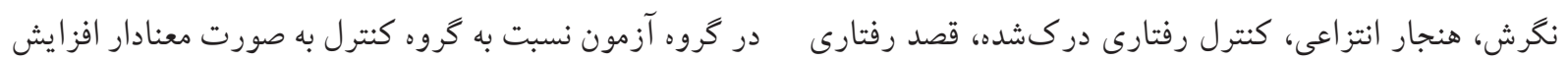

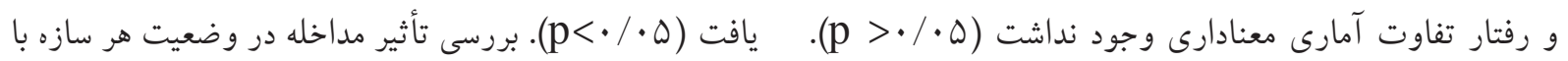


آزمون تىزوجى نشان داد تفاوت ميانگين نمره در گروه آزمون در گروه كنترل در هيجّدام از سازهها تفاوت آمارى معنادار

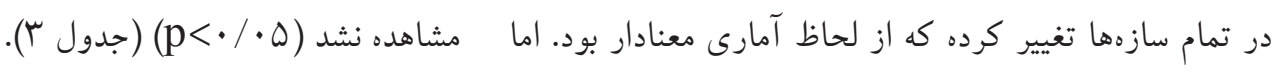

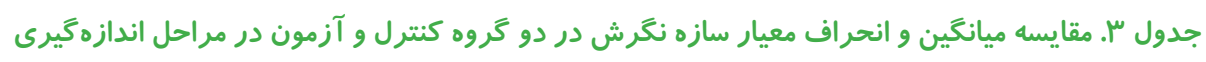

\begin{tabular}{|c|c|c|c|c|c|c|}
\hline \multirow{2}{*}{ P-value } & \multicolumn{2}{|c|}{ كروه كنترل } & \multicolumn{2}{|c|}{ كروه آزمون } & \multirow[t]{2}{*}{ مرحله } & \multirow{2}{*}{ سازه } \\
\hline & انحراف معيار & ميانگين & انحراف معيار & ميانگين & & \\
\hline$\cdot / r \Delta^{a}$ & $r / \mathcal{T} \Delta$ & $I V / F r$ & $F / 94$ & $1 N / 14$ & قبل از مداخله & \multirow{3}{*}{ 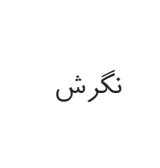 } \\
\hline$\cdot / \cdots 1^{a}$ & $r / v V$ & $1 V / 9 r$ & G/ST & $r 1 / 9 r$ & بعد از مداخله & \\
\hline- & \multicolumn{2}{|c|}{$\cdot / \wedge \cdot b$} & \multicolumn{2}{|c|}{$\cdot / \cdots 1^{b}$} & P-value & \\
\hline$\cdot|r|^{a}$ & $\Gamma / \tau$. & $19 / 19$ & $r / s r$ & $19 /$ H人 & قبل از مداخله & \\
\hline$\cdot / \cdots 1^{a}$ & $r / s q$ & $r \cdot / r q$ & $\Delta / \mu$ & $r Y / G G$ & بعد از مداخله & هنجار انتزاعى \\
\hline- & \multicolumn{2}{|c|}{$\cdot / \mu \Lambda^{b}$} & \multicolumn{2}{|c|}{$\cdot / \cdot 1^{b}$} & P-value & \\
\hline . / rua & $F / F r$ & IV/Ar & $r / 9 r$ & $I V / \wedge D$ & قبل از مداخله & \\
\hline$\cdot / \cdot 1^{a}$ & $0 / \cdot 1$ & $|N| \cdot F$ & G/Ir & $r \cdot|s|$ & بعد از مداخله & كنترل رفتارى \\
\hline- & \multicolumn{2}{|c|}{$\cdot / 18^{b}$} & \multicolumn{2}{|c|}{$\cdot / \cdot 1^{b}$} & P-value & \\
\hline.$/ 11^{a}$ & $r / \Delta \Delta$ & $11 / 94$ & $r / g r$ & $\mid r / F r$ & قبل از مداخله & \\
\hline$\cdot 1 \cdot 1^{a}$ & $r / \cdot \Lambda$ & Ir/AV & $9 / 90$ & $10 / F F$ & بعد از مداخله & قصد رفتارى \\
\hline - & \multicolumn{2}{|c|}{$\cdot / r \wedge^{b}$} & \multicolumn{2}{|c|}{$\cdot / \cdot 1^{b}$} & P-value & \\
\hline$\cdot /$ r $^{\mathrm{a}}$ & $r / \varsigma \Lambda$ & $\mid r / 9 \Lambda$ & $p / \mu r$ & $\mid r / F$. & قبل از مداخله & \\
\hline$\cdot / \cdot 1^{a}$ & $\Delta / \Delta \Lambda$ & $|r /| F$ & $s / F V$ & $19 /$ HA & بعد از مداخله & رفتار \\
\hline- & \multicolumn{2}{|c|}{$\cdot / r \Delta^{b}$} & \multicolumn{2}{|c|}{$\cdot / \cdot 1^{b}$} & P-value & \\
\hline
\end{tabular}

a: Independent T-test b: Paired Samples Test

بحث و نتيجاه گيرى

نتايج توصيفى مطالعه بيانكر اين موضوع بود كه بيشتر از ايدز در گروه آزمون بهبود يافت، در حاليكه اين ميزان در گروه

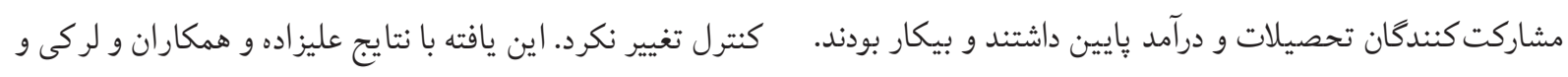

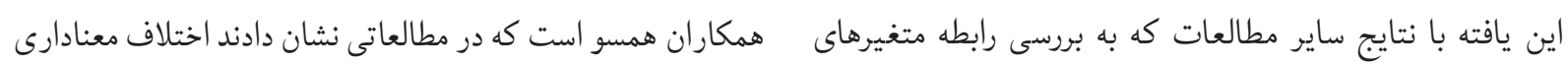

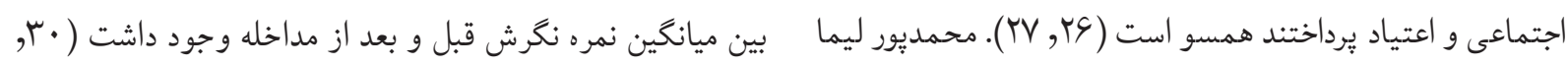

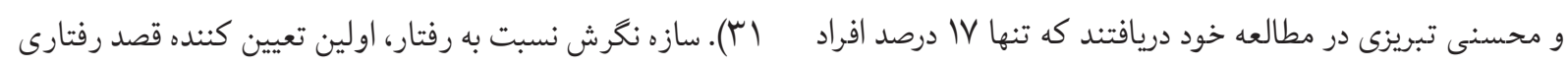

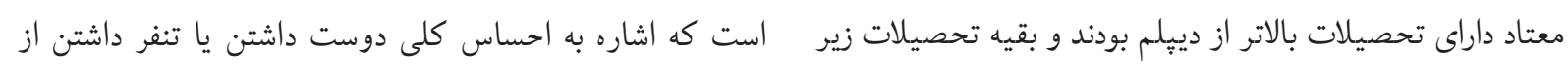

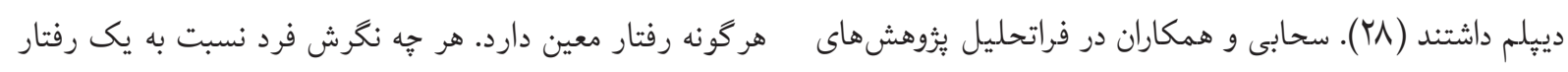

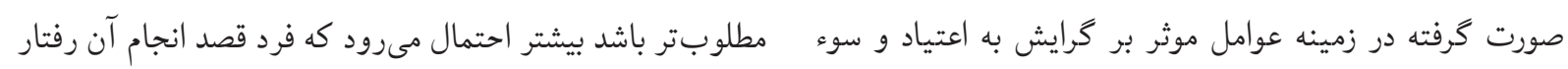

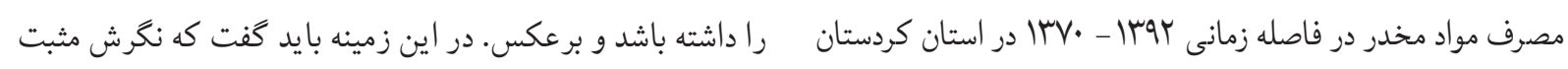

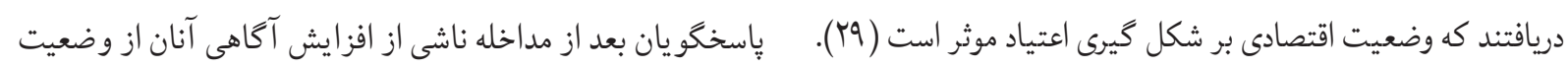

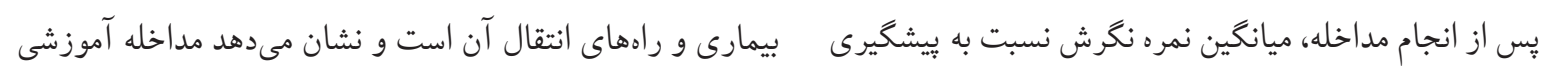


اختلاف معنادارى از لحاظ سازه قصد رفتارى وجود نداشت، اما

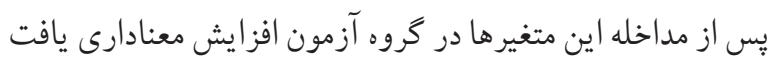

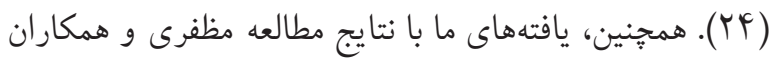

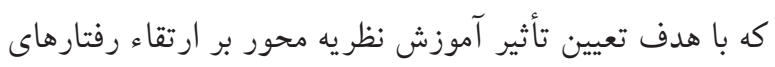
بيشكيرى كننده مادران در زمينه مصرف خودسر انه دارو در كود كان

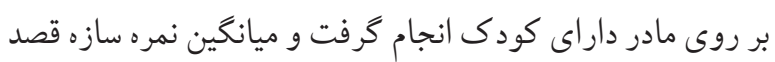

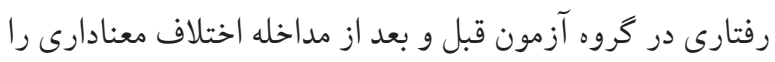

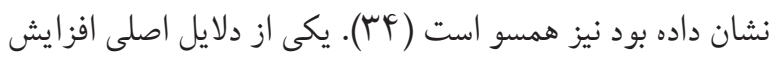

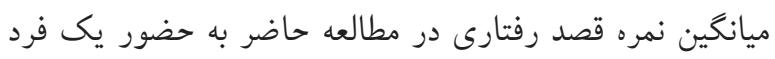

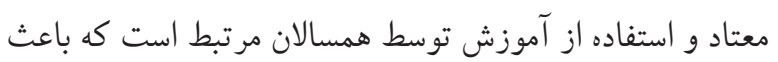
شده قصد افراد براى ييشخيرى تقويت شود.

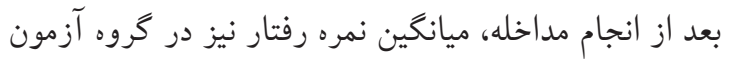
افزايش يافت. همسو با يافتهاى ما، مطالعه صادقى و همكاران با ميا

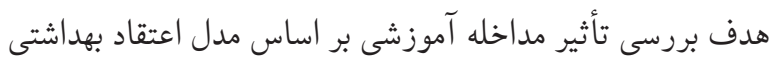

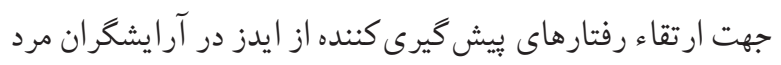

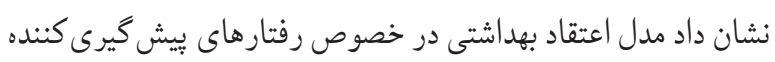

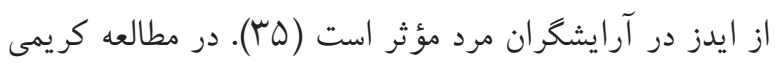

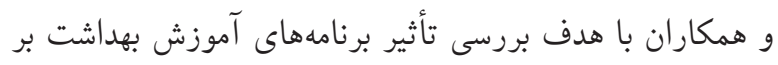

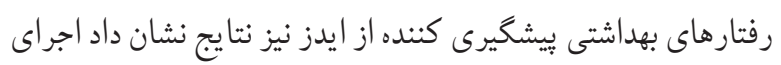
آموزش موجب افزايش عملكرد ييشخيرى از بيمارى ايدز در افراد

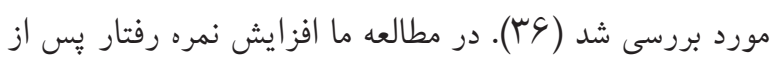

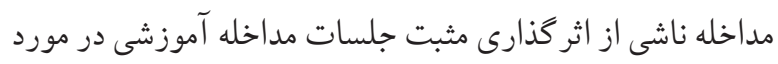
رفتارهاى بيشخيرى كننده از ايدز بوده است و نشان مى مدهد مداخله باعث شده رفتار بيشكيرى كننده در افر اد مورد مطالعه تقويت شود. مطالعه حاضر نيز همانند ساير مطالعات با محدوديتهايى رئي

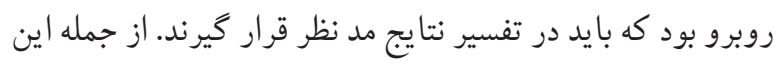

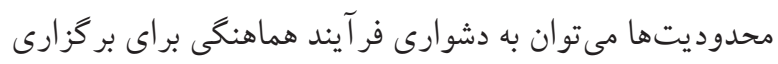

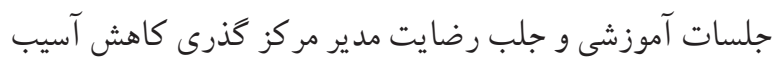

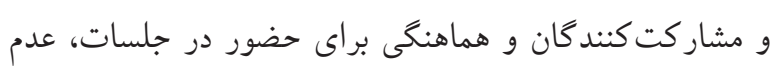

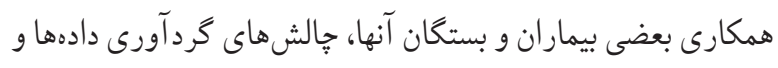

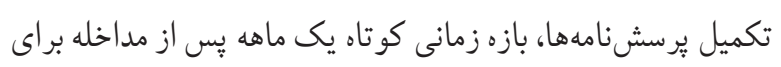

تاثير گذار بوده و مىتواند در رفتارهاى بيشگيرانه از بيمارىهايى نظير آج آى وى / ايدز موثر باشد.

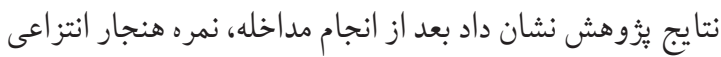

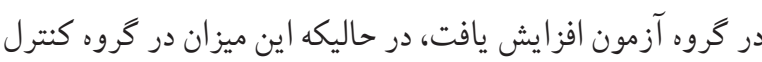

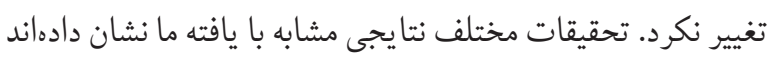

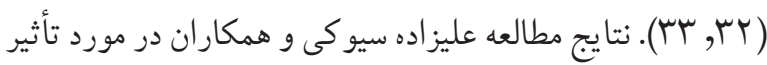

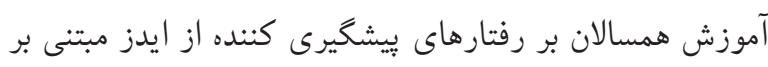

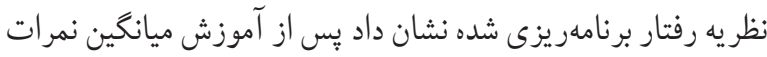

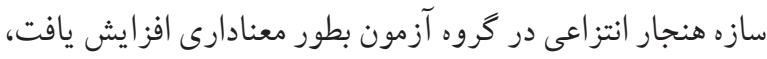

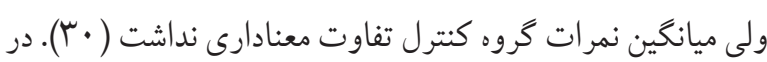
مطالعه حاضر به نظر مىرسد حضور يك فرد داراى تجارب مشابه و

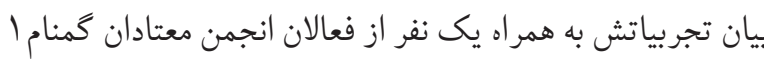

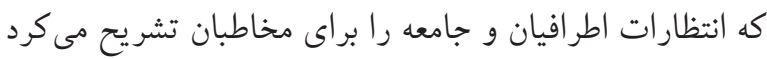
در بالا بردن نمره هنجارهاى انتزاعى مؤثر بوده است.

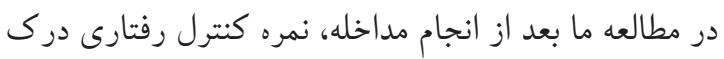

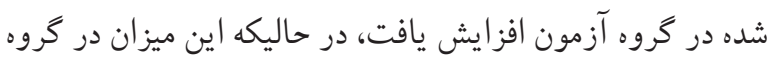

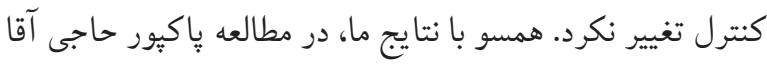

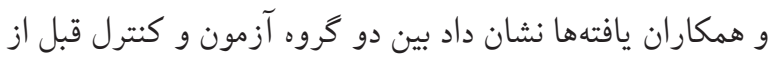

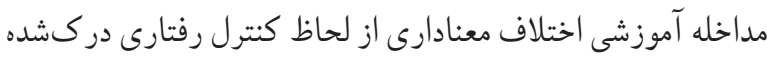

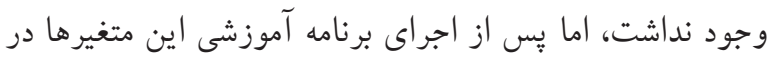

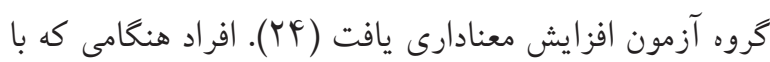
اثرات مثبت كنترل بر رفتار آشنا باشند و ويامدهاى مثبت ناشى از

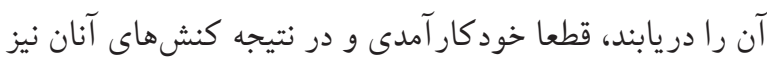
به سمت انجام رفتارهاى سالم و بهداشتى خواهد رفت. افر اد مورد

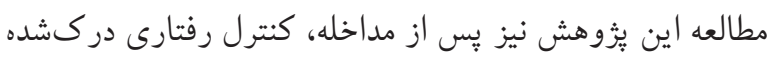

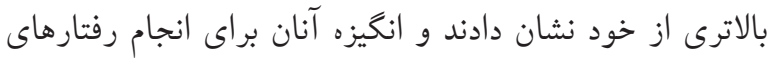
يبكشيرى كننده از ايدز بالاتر بود.

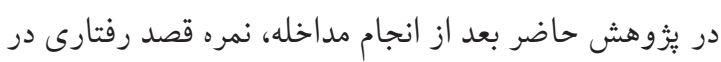
كروه آزمون افزايش يافت، در حاليكه اين ميزان در خروه كنترل تغيير نكرد. اين يافته همسو با نتايج ساير مطالعات است. مثلا، در

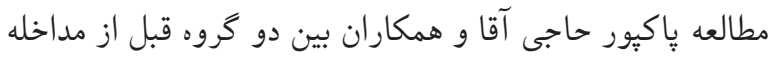


يِيشخيرى كننده از ايدز در معتادان مى شود. از اينرو، با توجه به تأثير معنادار آموزش انجام شده بر همه سازههاى نظريه رفتار برنامهريزى شده، آموزش مبتنى بر اين نظريه مى تواند به عنوان جارجوبى جهت طراحى و اجراى مداخلات آموزشى جهت بيشخيرى از ابتلا به ايدز در بين معتادان استفاده شود. در اين راستا و با توجه به افزايش نمره سازههاى نظريه، بايد در راستاى افزايش و تقويت نگرش مثبت و كنترل رفتارى درك شده معتادين در زمينه ييشخيرى از ايدز با آموزش مناسب تلاش نمود تا بدين وسيله ميزان رفتارهاى پرخطر كاهش يافته و قصد و رفتار مثبت ييشخيرانه آنها افزايش يابد و كنترل اين بيمارى به حداكثر برسد. تعارض در مناف نويسندگان اظهار مىدارند هيج گونه تعارض منافعى در مورد اين مقاله وجود ندارد

\section{References}

1. Siuki HA, Peyman N, Vahedian-Shahroodi M, Gholian-Aval $M$, Tehrani $H$. Health education intervention on HIV/ AIDS prevention behaviors among health volunteers in healthcare centers: An applying the theory of planned behavior.Journal of Social Service Research. 2019;45(4):582 https://doi.org/10.1080/01488376.2018.1481177

2. Tavoosi A, Zaferani A, Enzevaei A, Tajik P, Ahmadinezhad Z. Knowledge and attitude towards HIV/AIDS among Iranian students. BMC public health. 2004;4(1):17. https://doi.org/10.1186/1471-2458-4-17 PMid:15157281 PMCid:PMC420470

3. Gerbi GB, Habtemariam T, Robnett V, Nganwa D, Tameru B. Psychosocial factors as predictors of HIV/AIDS risky behaviors among people living with HIV/AIDS. Journal of AIDS and HIV research (Online). 2012;4(1):8. https: / / d o i.org / 10.5897 / JAHR 11.057 PMid:22374351

4. Kempton J, Hill A, Levi JA, Heath K, Pozniak A. Most new HIV infections, vertical transmissions and AIDS-related deaths occur in lower-prevalence countries. Journal of Virus Eradication. 2019;5(2):92. https://doi.org/10.1016/S2055-6640(20)30058-3

5. Asgharinekah SM, Sharifi F, Amel Barez M. The Need of Family-Based Sexual Education: A Systematic Review. Journal of Health Literacy. 2019;4(3):25-37.
ييخيرى و تكميل تيرسشنامها و تغيير در برخى جلسات آموزشى اشاره كرد. بر اساس نتايج مطالعه ييشنهاد مى گردد كه نظريه رفتار برنامهريزى شده در طراحى برنامههاى تغيير رفتار سلامتى بيشتر مد نظر قرار گيرد. هم جنين، در مورد معتادان مواد مخدر، مداخله با دورههاى يُخيرى طولانى تر و ماهه و بيشتر اجرا كردد. هم جنين، ارائه آموزش در قالب بر گزارى كار گاههاى آموزشى، جلسات آموزشى و سمينارهاى علمى، تمر كز بر ارائه خدمات و ارائه وسايل يِيشخيرى از جمله كاندوم به صورت رايگان، و يا يش مداوم وضعيت اج آى آى وى / ايدز در بين معتادان براى برنامهريزىهاى مناسب مىتواند مد نظر قرار كيرد. نتيجاه كيرى

يافتهاى مطالعه حاضر نشان داد مداخله آموزشى با نظريه رفتار برنامهريزىشده باعث بهبود و تقويت نگرش، افزايش كنترل رفتارى دركشده، ايجاد هنجارهاى انتزاعى و شكل گيرى قصد و رفتارى

6. Rafiei N, Aghapoor S, Behnampour N, Heshmati $\mathrm{H}$, Ghasemyani S. Promoting AIDS Preventive Beliefs in Turkmen Students by Using the Health Belief Model in Aq-Qala. Iranian Journal of Health Education and Health Promotion. 2019;7(4):323-32. https://doi.org/10.29252/ijhehp.7.4.323

7. Control CfD, Prevention. The global HIV/AIDS pandemic, 2006. MMWR Morbidity and mortality weekly report. 2006;55(31):841-4.

8. Hurst T. World drug report. The encyclopedia of women and crime. 2019:1-2. https://doi.org/10.1002/9781118929803.ewac0543 PMCid:PMC6938603

9. Pandey A, Galvani AP. The global burden of HIV and prospects for control. The Lancet HIV. 2019;6(12):e809 https://doi.org/10.1016/S2352-3018(19)30230-9

10. Shojaeizadeh D, Ebrahim Taheri G, Tehrani H, Hosseini $\mathrm{SH}$. The effect of education on knowledge and attitude of high school students about AIDS in Faruj, Iran. Health and Development Journal. 2020;1(1):67-73.

11. Majdi M, Khani $H$, Azadmarzabadi E, Montazeri A, Hallajian E, Babamahmodi $F$, et al. Knowledge, attitudes and practices towards HIV/AIDS among Iranian prisoners in Mazandaran province in the south-coast area of the Caspian Sea. EMHJ-Eastern Mediterranean 
Health Journal, 17 (12), 904-910, 2011. 2011 https://doi.org/10.26719/2011.17.12.904 PMid:22355942

12. Avanessian E, Naserirad M, Abrahamian H, Anis S. Size of Social Network and Probability of Occurrence of HIV/ AIDS among Sexually Affected Patients in Behavioral Diseases Consulting Centers. Iranian Journal of Health Education and Health Promotion. 2017;5(2):73-80. https://doi.org/10.30699/ihepsa.journal.5.2.1

13. Lotfi R, Tehrani FR, Yaghmaei F, Hajizadeh E. Barriers to condom use among women at risk of HIV/AIDS: a qualitative study from Iran. BMC women's health. 2012;12(1):1-10. https://doi.org/10.1186/1472-6874-12-13 PMid:22624530 PMCid:PMC3519504

14. Rahimi-Movaghar A, Amin-Esmaeili M, Haghdoost A-a, Sadeghirad B, Mohraz M. HIV prevalence amongst injecting drug users in Iran: a systematic review of studies conducted during the decade 1998-2007. International Journal of Drug Policy. 2012;23(4):271-8. https://doi.org/10.1016/j.drugpo.2011.09.002 PMid:22000694

15. SeyedAlinaghi SA, Farhoudi B, Mohraz M, Pahlaviani FG, Hosseini M, Farnia M, et al. Prevalence and correlates of HIV infection among male prisoners in Tehran, Iran. Archives of Iranian Medicine. 2017;20(6):356-60.

16. Falk DE, Yi H-y, Hilton ME. Age of onset and temporal sequencing of lifetime DSM-IV alcohol use disorders relative to comorbid mood and anxiety disorders. Drug and alcohol dependence. 2008;94(1-3):234-45. https://doi.org/10.1016/j.drugalcdep.2007.11.022 PMid:18215474 PMCid:PMC2386955

17. Zhang Y, Shan H, Trizzino J, Ruan Y, Beauchamp G, Mâsse $B$, et al. Demographic characteristics and risk behaviors associated with HIV positive injecting drug users in Xinjiang, China. Journal of Infection. 2007;54(3):285-90. https://doi.org/10.1016/j.jinf.2006.06.001 PMid:16831464

18. B. F. Epidemic of HIV/AIDS, injection addiction, harm reduction and syringe \& needle program. Tehran; Seda center publication. 2003.

19. Jürgens R, Ball A, Verster A. Interventions to reduce HIV transmission related to injecting drug use in prison. The Lancet infectious diseases. 2009;9(1):57-66. https://doi.org/10.1016/S1473-3099(08)70305-0

20. Chrissie P. Impacts of a Peer-Group Intervention on HIV Related Knowledge, Attitudes, and Personal Behaviors for Urban Hospital Workers in Malawi. Journal of the Association of Nurses in AIDs Care. 2009;20:203-42. https://doi.org/10.1016/j.jana.2008.12.005 PMid:19427600 PMCid:PMC4177099

21. brahimi D DS, Harrison, s Infectious Diseases1994. Tehran: Hayyan cultural Institute Pub. 2000.

22. Cohen MS, Hellmann N, Levy JA, DeCock K, Lange J. The spread, treatment, and prevention of HIV1: evolution of a global pandemic. The Journal of clinical investigation. 2008;118(4):1244-54. h tt p s: / / d o i.org / 10.1172 / J CI 34706 PMid:18382737 PMCid:PMC2276790

23. Armitage CJ, Conner M. Efficacy of the theory of planned behaviour: A meta-analytic review. British journal of social psychology. 2001;40(4):471-99. https://doi.org/10.1348/014466601164939 PMid:11795063

24. ME PHA, Mohammadi B. Impact theory of planned behavior as skills-based education for HIV prevention in adolescents. Iranian Journal of Nursing. 2013;25(78):1-13.

25. Gebreeyesus Hadera $H$, Boer $H$, Kuiper WA. Using the theory of planned behaviour to understand the motivation to learn about HIV/AIDS prevention among adolescents in Tigray, Ethiopia. AIDs care. 2007;19(7):895-900. https://doi.org/10.1080/09540120701203311 PMid:17712693

26. Nemati F, Shahmoradi S, Rahimi FS. Investigating the Factors Affecting Drug Abuse and Addiction with a Grounded Theoretical Approach. Scientific Quarterly Research on Addiction. 2020;14(57):11-34. https://doi.org/10.29252/etiadpajohi.14.57.11

27. Abdeli Saj, Amani Sj. Effective factors in youth drug abuse in Iran: Analysis of fuzzy cognitive maps based on expert opinion. Scientific Quarterly Research on Addiction. 2016;10(39):195-215 .

28. Mohammadpoor Lima H, Mohseni Tabrizi Ar. A Sociological Explanation of Effective Factors on Tendency Toward Risky Behavior in the Rural Areas of Guilan province (Case Study: Drug Abuse and Addiction in the Eshkevar Rural Areas, RahimAbad District of Rudsar). Journal of Social Problems of Iran. 2018;8(2):191-207 .

29. Sahabi J, mafakheri bashmagh J, soltanian S, Shapari R, Aghabeigpouri $\mathrm{H}$. A meta-analysis of research conducted on factors affecting disposition to addiction and drug abuse and preventive measures taken over 1991-2013 in Kurdistan Province with a focus on behavioral and social factors. Two Quarterly Journal of Contemporary Sociological Research. 2016;5(8):99-122 .

30. Alizadeh Seivaki H Zl, Rakhshani F,. The impact of peer education on HIV preventive behaviors based on the 
theory of planned behavior in second high school students in Zahedan. J Ofogh Danesh. 2013;18(5):233-40.

31. Loraci M, Taffazoli M. Latifnejad roudsari R. The Effect of a Training program Based on the Theory of Planned Behavior (TPB) on Sexual High Risk Behavioral Intentions in female Prisonors, Vakil Abad prison, Mashhad. Iran J Kerman Univ Med Sci. 2016;23(4):497-514.

32. Baghianimoghadam M, Tavakoli B, Ayatollahi J, Mirzaei M. The effect of education based on the theory of planned behavior on preventive behaviors of cutaneous Leishmaniasis in mothers living in endemic city of Natanz. Tolooebehdasht. 2016;15(3):54-66 .

33. Mokarrami E, Jalili Z, Tavakoli Ghouchani H. The Effect of Education Based on the Theory of Planned Behavior on Preventive Behaviors of Vaginitis in Health Ambassadors. Iranian Journal of Health
Education and Health Promotion. 2019;7(2):211-9. https://doi.org/10.30699/ijhehp.7.2.211

34. Mozafari S, Shamsi M, Roozbahani N, Ranjbaran M. Measurement of Theory Structures of Planned Behavior in Prevention of Self-Treatment in Mothers of Azadshahr City. Sci J Hamadan Nurs Midwifery Fac. 2012;23(2):15-23.

35. Sadeghi R, Mazloomy S, Hashemi M, Rezaeian M. The effects of an educational intervention based on the health belief model to enhance HIV-preventive behaviors among male barbers in Sirjan. Journal of Rafsanjan University of Medical Sciences. 2016;15(3):235-46.

36. Karimi M, Ghofranipor F, Heidarnia A. The Effect of Health Education Based on Health Belief Model on Preventive Actions of AIDS on Addict in Zarandieh. Journal of Guilan University of Medical Sciences. 2009;18(70):64-73. 